Revue Française de Civilisation Britannique

\title{
Compte-rendu de Beyond Brexit: Towards a British Constitution (2019), Vernon Bogdanor
}

\section{Stéphanie Bory}

\section{(2) OpenEdition}

\section{Journals}

Édition électronique

URL : http://journals.openedition.org/rfcb/5489

DOI : $10.4000 /$ rfcb.5489

ISSN : 2429-4373

Éditeur

CRECIB - Centre de recherche et d'études en civilisation britannique

Référence électronique

Stéphanie Bory, "Compte-rendu de Beyond Brexit: Towards a British Constitution (2019), Vernon Bogdanor », Revue Française de Civilisation Britannique [En ligne], XXV-2 | 2020, mis en ligne le 25 mai 2020, consulté le 27 mai 2020. URL : http://journals.openedition.org/rfcb/5489 ; DOI : https://doi.org/ $10.4000 /$ rfcb.5489

Ce document a été généré automatiquement le 27 mai 2020.

\section{(†)

Revue française de civilisation britannique est mis à disposition selon les termes de la licence Creative Commons Attribution - Pas d'Utilisation Commerciale - Pas de Modification 4.0 International. 


\title{
Compte-rendu de Beyond Brexit: Towards a British Constitution (2019), Vernon Bogdanor
}

\author{
Stéphanie Bory
}

\section{RÉFÉRENCE}

Vernon Bogdanor, Beyond Brexit: Towards a British Constitution, Londres, I.B. Tauris, 2019, 304 pages

1 Né en 1943, diplômé d'Oxford en 1964 en philosophie, économie et politique, Vernon Bogdanor, spécialiste en histoire constitutionnelle, est Professeur au Centre pour la politique et le gouvernement britanniques (Centre for British Politics and Government) de King's College, à Londres, après avoir enseigné jusqu'en 2010 au Brasenose College à Oxford. Professeur émérite à Gresham College, il donne régulièrement des conférences sur la politique et l'histoire britanniques, conférences disponibles sur le site de l'université. Il a eu, dans sa carrière, des étudiants qui ont par la suite occupé le devant de la scène, comme David Cameron, Premier ministre de 2010 à 2016 qu'il a décrit comme "a moderate and sensible Conservative ${ }^{1}$, Camilla Cavendish, ancienne directrice politique de David Cameron et membre de la Chambre des Lords, ou Katherine Allen, directrice depuis 2000 de Amnesty International UK.

Régulièrement invité sur les chaînes de radio et de télévision, il est l'auteur de plus d'une dizaine d'ouvrages, tous reconnus, sur le gouvernement et la politique, dont The People and the Party System: The Referendum and Electoral Reforms in British Politics (1981), The Monarchy and the Constitution (1997), Power and the People: A Guide to Constitutional Reform (1997) et The Coalition and the Constitution (2011), ainsi que de très nombreux articles. Il a été et est également conseiller auprès du gouvernement et de plusieurs commissions parlementaires, comme le Select Committee on the European Communities de la Chambre des Lords (1982-1983), ainsi qu'auprès de différents gouvernements 
étrangers, en République tchèque, Israël, Hongrie ou Slovaquie, sur les réformes électorales et constitutionnelles en tant que membre de délégations du Conseil de l'Europe et de la American Bar Association. Vernon Bogdanor est membre de la British Academy et membre honoraire du Institute of Advanced Legal Studies. Il a été nommé Commander of the Order of the British Empire (CBE) en 1998 et Chevalier de la Légion d'Honneur en 2009 par Nicolas Sarkozy pour son travail sur le droit et l'histoire politique de la Grande-Bretagne et de la France. Il est aujourd'hui unanimement considéré comme «l'un des meilleurs experts en droit constitutionnel au RoyaumeUni $»^{2}$.

3 Vernon Bogdanor s'est récemment beaucoup intéressé au modèle constitutionnel britannique, en particulier dans le contexte de la dévolution et des réformes constitutionnelles introduites par le gouvernement Blair depuis 1997 - sujet de son ouvrage The New British Constitution (2009) - et du vote pour le Brexit en 2016, objet de son dernier ouvrage, Beyond Brexit: Towards a British Constitution, publié en février 2019 chez Bloomsbury. Celui-ci comporte à l'intérieur de l'ouvrage un titre différent, Beyond Brexit: Britain's Unprotected Constitution. Bogdanor indique dans les Remerciements que le chapitre 5, "Europe and the Rights of the Citizen", reprend largement un pamphlet publié en 2018 pour la Constitution Society, d'où les deux titres. Il ajoute que, selon lui, les débats sur le Brexit ont essentiellement porté sur l'impact économique de la sortie du Royaume-Uni de l'Union européenne, mais ont négligé un aspect pourtant primordial, à savoir l'effet considérable qu'elle aura sur la Constitution britannique, notamment le Parlement, le pouvoir juridique et les droits individuels. Il s'interroge sur la capacité du Royaume-Uni à résister aux forces centrifuges et centripètes libérées par le Brexit. Il explique enfin que le postulat de départ de l'ouvrage lui a été suggéré par Andrew Blick, Directeur du Centre for British Politics and Government et Professeur en politique et histoire contemporaine à King's College, selon lequel le Brexit constitue l'événement constitutionnel ("a constitutional moment $»^{3}$ ) le plus important au Royaume-Uni depuis la Restauration de la monarchie en 1660 qui conduit, moins de 30 ans plus tard, à la Glorieuse Révolution. En effet, pour la première fois, le Parlement britannique est tenu de voter et mettre en œuvre une décision à laquelle il est en majorité opposé, à la suite d'un référendum consultatif que les leaders des principaux partis se sont engagés à respecter. Ce qui est source de conflit entre le principe au cœur de la Constitution britannique, la souveraineté parlementaire, définie notamment par A.V. Dicey au XIX ${ }^{e}$ siècle, et la souveraineté populaire. L'auteur insiste sur le fait qu'une sortie de l'Union européenne ne rendra pas au Parlement sa souveraineté puisqu'il n'y aura clairement pas de retour au cadre politique et constitutionnel de 1972, date de l'adoption par le Parlement du European Communities Act : « Too much will have changed since 1973 ; in any case, the Britain of 2019 is a very different place from the Britain of 1973. $»^{4}$

4 L'intérêt de cet ouvrage est multiple. Vernon Bogdanor aborde et manipule tout d'abord des concepts politiques et juridique complexes mais en faisant preuve d'une grande pédagogie et une infinie clarté. De plus, la progression des différents chapitres, au nombre de sept, est extrêmement logique et cohérente dans la mesure où il développe un point précis par chapitre, dans une relation de causalité avec le chapitre suivant. Tout comme ses publications précédentes, ce dernier opus constitue une lecture indispensable à quiconque s'intéresse au Brexit et à son impact au RoyaumeUni. 
Bogdanor explore dans le premier chapitre intitulé «Britain and Europe: The Poisoned Chalice» les relations difficiles entre le Royaume-Uni et la Communauté économique européenne (CEE), puis l'Union européenne (UE), depuis les années 1950 qui marquent le développement du concept originel d'unité européenne. Il revient sur les différentes étapes de l'entrée chaotique du Royaume-Uni dans la CEE - la première candidature en 1961 et le véto du Général de Gaulle deux ans plus tard, la deuxième candidature en 1967 également rejetée et l'entrée finalement en 1973 - malgré une réelle implication du Royaume-Uni dans les affaires européennes par le biais de signature d'accords économiques et commerciaux et sa contribution majeure dans la création du Conseil de l'Europe. S'appuyant sur les travaux de A.V. Dicey et Sidney Low, il définit la Constitution britannique avant l'entrée dans la CEE, opposant souveraineté parlementaire et souveraineté populaire. Selon Bogdanor, les systèmes constitutionnels britannique et européen étaient bien trop différents pour être totalement compatibles et l'impact de l'appartenance à la CEE sur la souveraineté du Parlement britannique n'a pas suffisamment été débattu au moment de l'adhésion.

6 Dans le chapitre suivant, "Europe and the Sovereignty of Parliament", l'auteur présente plusieurs décisions de justice majeures prises par la Cour européenne depuis les années 1960 qui ont, de façon graduelle, limité la souveraineté du Parlement britannique après 1973 et introduit le principe de «judicial review » par l'adoption de deux principes fondamentaux, la doctrine de l'effet direct et celle de la primauté du droit communautaire sur le droit national des états membres. Bogdanor termine ce deuxième chapitre sur le paradoxe du European Union Act 2011 introduit par David Cameron: tout en statuant que le Parlement est souverain, cette loi oblige tout parlement à venir à consulter les électeurs par référendum pour tout nouveau transfert de pouvoirs à l'UE.

7 Il poursuit son raisonnement en étudiant, dans le chapitre "Europe and the Referendum ", comment l'appartenance du Royaume-Uni à la CEE, puis à l'UE, a engendré une révolution constitutionnelle supplémentaire: l'introduction du référendum dans la vie politique britannique, innovation jusqu'alors considérée comme anticonstitutionnelle et jamais utilisée à l'échelle de tout le Royaume-Uni jusqu'en juin 1975, date du référendum organisé par Harold Wilson pour des raisons essentiellement tactiques et politiques. Bogdanor rappelle que cette consultation, qui ne devait constituer un précédent, a pourtant ouvert la voie à de multiples référendums, tant régionaux que nationaux, le dernier étant celui organisé en juin 2016 par David Cameron, pour les mêmes raisons que Wilson plus de quarante ans plus tôt.

Cette réflexion sur une telle innovation constitutionnelle conduit l'auteur à se pencher, dans le chapitre suivant « Europe and the Collective Responsibility of Ministers », sur la décision alors prise pour la première fois par un gouvernement majoritaire de suspendre la convention clairement établie depuis le XIX ${ }^{e}$ siècle et définissant le principe de la responsabilité collective des membres du gouvernement. En effet, le Premier ministre Harold Wilson décide en 1975 de lever cette convention le temps de la campagne référendaire, tout comme le gouvernement de coalition entre 2010 et 2015, puis David Cameron pour le référendum sur le Brexit en 2016.

9 Dans le chapitre 5, «Europe and the Rights of the Citizens ", mentionné précédemment, Bogdanor considère que le Brexit va profondément réduire les droits des Britanniques qui ne bénéficieront plus de la protection de la Charte européenne des droits fondamentaux, adoptée en 2000 et incluse dans le Traité de Lisbonne de 2008, tout en 
augmentant considérablement le pouvoir non pas du Parlement mais du gouvernement. Pour remédier à cette situation, il encourage l'adoption d'une Bill of Rights britannique.

S'interrogeant dans le chapitre 6 «Brexit and Devolution: The Future of the United Kingdom» sur l'avenir de l'Union britannique dans un contexte de tensions renouvelées et accrues entre le pouvoir central et les administrations dévolues, l'auteur revient sur les concepts d'état unitaire et d'état union. Il insiste en préambule sur le fait que, depuis 1998 et l'introduction de la dévolution, le Royaume-Uni est devenu un état multinational. Il étudie le processus de dévolution et l'impact du Brexit pour chaque nation constitutive de l'Union britannique, l'Ecosse, le pays de Galles, l'Irlande du Nord - tout en élargissant sa réflexion à la République d'Irlande - et l'Angleterre, " anomalie " selon lui dans les accords de dévolution. Il aborde ainsi dans ce chapitre différents points comme le référendum écossais sur l'indépendance, la question dite EVEL («English Votes for English Laws»), la convention Sewel et la question épineuse des accords financiers entre Londres et les administrations dévolues post-Brexit. Bogdanor conclue sur l'idée que l'avenir de l'Union britannique est incertain en partie en raison des quatre interprétations différentes de la Constitution qui prévalent aujourd'hui dans chaque nation britannique.

11 C'est la raison pour laquelle il termine son ouvrage par un dernier chapitre, «Brexit: A Constitutional Moment ", dans lequel il insiste sur la nécessité pour le Royaume-Uni d'adopter une constitution codifiée, seul moyen, selon lui, de lever les nombreuses incertitudes constitutionnelles engendrées par l'appartenance à l'UE, telles l'utilisation du référendum, la définition claire des droits des citoyens ou la relation entre Westminster et les administrations dévolues. Le Brexit a en effet révélé à quel point la Constitution britannique est sans protection, tout en offrant l'occasion d'y remédier, ce que souligne Bogdanor dans les dernières lignes de son ouvrage :

It is just possible that we are now approaching a peaceful constitutional moment, one marked not by revolution or a struggle for independence, but by the concatenation of interconnected constitutional problems, all pressing insistently for resolution. It is just possible that Brexit will prove to be that very break in continuity that will herald our own constitutional moment. ${ }^{6}$

\section{NOTES}

1. Vernon Bogdanor, cité dans Brian Wheeler, "The David Cameron Story", BBC News, 06-12-2005, http://news.bbc.co.uk/2/hi/uk_news/politics/4502656.stm, consulté en avril 2020.

2. Voir Sonia Delesalle-Stolper, "Vernon Bogdanor : 'Le Parlement est entravé par le peuple' ", Libération,

02-04-2019, https://www.liberation.fr/planete/2019/04/02/vernon-bogdanor-le-parlement-estentrave-par-le-peuple_1719020, consulté en avril 2020.

3. Vernon Bogdanor, Beyond Brexit: Towards a British Constitution, Londres, I.B. Tauris, 2019, p. vi.

4. Ibid., p. 1.

5. Ibid., p. 198. 
6. Ibid., p. 278. 